\title{
Ad5-spike COVID-19 vaccine does not aggravate heart damage after ischemic injury
}

\section{Shanshan Gu}

Sun Yat-sen University

\section{Zhongyan Chen}

Sun Yat-sen University

Xiangfu Meng

Hubei University

Ge Liu

Sun Yat-Sen University

$\mathrm{HeXu}$

Sun Yat-Sen University

Linwei Wu

Sun Yat-Sen University

Jixing Gong

Hubei University

Ding Chen

Sun Yat-Sen University

Bingqing Xue

Hubei University

Liying Huang

Sun Yat-Sen University

Lihang Zhu

Hubei University

Zhongjun Wan

Hubei University

Xiaolong Cai

GeneMedi Suzhou Biotechnology

Xiaoyan Zhang

GeneMedi Suzhou Biotechnology

Jia Wang

Sun Yat-Sen University

Donghui Zhang

Hubei University

Nan Cao ( $\nabla$ caon3@mail.sysu.edu.cn ) 
Sun Yat-sen University https://orcid.org/0000-0002-1660-4728

\section{Research Article}

Keywords: COVID-19, spike protein, host receptor ACE2, heart disease, SARS-CoV-2

Posted Date: June 7th, 2021

DOl: https://doi.org/10.21203/rs.3.rs-574304/v1

License: (c) (i) This work is licensed under a Creative Commons Attribution 4.0 International License. Read Full License 


\section{Ad5-spike COVID-19 vaccine does not aggravate heart damage after ischemic injury}

Shanshan $\mathrm{Gu}^{1,3, \uparrow}$, Zhongyan Chen ${ }^{1,3, \uparrow}$, Xiangfu Meng ${ }^{2, \uparrow}, \mathrm{Ge} \mathrm{Liu}^{1,3, \uparrow}, \mathrm{He} \mathrm{Xu}^{1,3, \uparrow}$, Linwei Wu ${ }^{1,3}$, Jixing Gong $^{2}$, Ding Chen ${ }^{1,3}$, Bingqing Xue ${ }^{2}$, Liying Huang ${ }^{1,3}$, Lihang Zhu ${ }^{2}$, Zhongjun Wan ${ }^{2}$, Xiaolong $\mathrm{Cai}^{4,5}$, Xiaoyan Zhang ${ }^{4,5}$, Jia Wang ${ }^{1,3}$, Donghui Zhang ${ }^{2, *}$, and Nan Cao ${ }^{1,3, *}$

${ }^{1}$ Zhongshan School of Medicine, Sun Yat-Sen University, Guangdong 510080, China.

${ }^{2}$ State Key Laboratory of Biocatalysis and Enzyme Engineering, Hubei Province Key Laboratory of Biotechnology of Chinese Traditional Medicine, National \& Local Joint Engineering Research Center of High-throughput Drug Screening Technology, Hubei University, Wuhan 430062, China

${ }^{3}$ Key Laboratory for Stem Cells and Tissue Engineering (Sun Yat-Sen University), Ministry of Education, Guangdong, China.

${ }^{4}$ GeneMedi Suzhou Biotechnology Co., Ltd, China.

${ }^{5}$ Hanbio Biotechnology Shanghai Co., Ltd, China

These authors contributed equally: Shanshan Gu, Zhongyan Chen, Xiangfu Meng, Ge Liu, and $\mathrm{He} \mathrm{Xu.}$

Correspondence: Donghui Zhang (dongh.zhang@hubu.edu.cn) or Nan Cao (caon3@mail.sysu.edu.cn) 


\section{Abstract}

Hopes for a COVID-19 vaccine are now a reality. The spike protein of SARS-CoV-2, which majorly binds to the host receptor ACE2 for cell entry, is used by most of the COVID-19 vaccine candidates as a choice of antigen. ACE2 is highly expressed in the heart and is known to be protective in multiple organs. Interaction of spike with ACE2 has been reported to reduce ACE2 expression and affect ACE2-mediated signal transduction in the heart. However, whether a spike-encoding vaccine will aggravate myocardial damage after a heart attack via affecting ACE2 remains unclear. Therefore, for patients with or at risk of heart diseases, questions arise around the safety of the spike-based vaccines. Here, we demonstrate that ACE2 is up-regulated and protective in the injured mouse heart after myocardial ischemia/reperfusion (I/R). Infecting human cardiomyocyte, smooth muscle cells, endothelial cells, and cardiac fibroblasts with a recombinant adenovirus type-5 vectored COVID-19 vaccine expressing the spike protein (AdSpike) does not affect cell survival and cardiomyocyte function, whether the cells are subjected to hypoxia-reoxygenation injury or not. This observation is further confirmed in human engineered heart tissues. Furthermore, AdSpike vaccination does not aggravate heart damage in wild-type or humanized ACE2 mice after I/R injury, even at a dose that is ten-fold higher as used in human. This study represents the first systematic evaluation of the safety of a leading COVID-19 vaccine under a disease context and may provide important information to ensure maximal protection from COVID-19 in patients with or at risk of heart diseases. 
The ongoing pandemic of coronavirus disease 19 (COVID-19) caused by a coronavirus SARSCoV-2 has spurred an unprecedented public health crisis worldwide. Hence, the development of a safe and effective vaccine that can prevent SARS-CoV-2 infection and transmission has rapidly become top priority. For cell entry, the SARS-CoV-2 virus majorly binds to the host receptor angiotensin-converting enzyme 2 (ACE2) through its spike glycoprotein, which is the only viral protein that interacts with host cells and is the most diverging protein between different coronaviruses [1]. Therefore, generating a vaccine encoding/introducing the spike protein is the strategy used by the majority of COVID-19 vaccine candidates, including vaccines based on viral vectors, nanoparticles/virus-like particles, proteins/peptides, RNA, and DNA [2]. There has been an unprecedented rapid response by vaccine developers with now over 60 COVID-19 vaccine candidates in clinical trials and ten having been approved for at least limited use as of 1 March 2021. Although trials of the approved spike-based COVID-19 vaccines have not detected vaccinerelated serious adverse events, it should be noticed that safety of these vaccines were mostly evaluated in healthy volunteers, and little is known about their effects on patients with or at risk of chronic diseases [3-5].

ACE2 is a membrane-localized aminopeptidase that is highly expressed in the heart and blood vessels and has direct effects on cardiac function and multiple organs via counter-regulation of the renin-angiotensin system (RAS), a primary cardiovascular regulatory system [6]. Increased ACE2 expression is observed after myocardial infarction in both rodent and human [7], suggesting that ACE2 may also be involved in regulating heart repair following ischemic injury. We have confirmed the significant increase of ACE2 in the injured mouse heart after myocardial ischemia/reperfusion (I/R) (Fig. 1A-B). Furthermore, we found that ACE2 overexpression reduced the infarct size and improved heart function during I/R, whereas ACE2 knockdown aggravated heart damage (Fig. 1C-F). These data suggest that ACE2 has a protective role during myocardial I/R. However, interaction of the spike protein with ACE2 during virus infection has been shown to reduce ACE2 expression [8,9] and alter the RAS signal transduction. For example, binding of spike to ACE2 upregulates the Ras-ERK-AP-1 pathway leading to activation of pro-remodeling 
factors such as the C-C motif chemokine ligand 2 (CCL2), which may contribute to cardiac injury and subsequently cause the fibrosis associated with disease manifestation [10].This raises a pivotal question: will the vaccine encoding the spike protein increase the risk of myocardial damage after a heart attack via binding to and affecting the cardiac ACE2? For patients with coronary or ischemic heart disease or people at high heart attack risk (e.g., arrhythmias, hypertension, or diabetes mellitus), questions arise around the safety after COVID-19 vaccinations in the setting of increased ACE2 expression in the injured heart and warrant close investigation. Thus, in this study, we evaluate the effects of spike-expressing vaccine on hearts that subject to I/R injury using human cardiac cells, engineered human heart tissues, and humanized ACE2 mice (Supplementary Fig. S1).

We firstly examined the ex vivo effect of the spike-based vaccine on the four major cell types of human heart (Supplementary Fig. S2A), using cardiomyocytes (hCMs) and smooth muscle cells (hSMCs) that were differentiated from human embryonic stem cells (Supplementary Fig. S2B-C), as well as primary human cardiac fibroblast (hCFs) and endothelial cells (hECs). A recombinant adenovirus type-5 (Ad5) vectored COVID-19 vaccine expressing the spike protein (AdSpike) was chosen as a proof-of-principle, because it has the longest expression period when compared with the vaccines that temporally delivering the spike DNA, mRNA, or recombinant protein, thus may have a theoretically maximum effect on the host tissues and cells. We fine-tuned the multiplicity of infection (MOI; i.e. the number of infectious viral particles per cell) of AdSpike to achieve either low (50-70\%), moderate ( 90\%), or high ( 100\%) expression efficiency of spike on each cell type (Supplementary Fig. S2D), and evaluated their effects on cell survival under normoxia condition or after hypoxia-reoxygenation (hyp-reox) injury. As expected, in all four cell types examined, normoxic cultured cells that received the control adenovirus (AdVector) or AdSpike had a comparable survival rate as revealed by the terminal-deoxynucleotidyl transferase mediated nick end labeling (TUNEL) (Supplementary Fig. S3A) and calcein-AM/propidium iodide (PI) staining (Supplementary Fig. S3B) analyses. Notably, AdSpike had no or minimal influence on cell survival in hCMs, hSMCs, hCFs, or hECs that underwent 12 hours of hypoxia and 12 hours 
of reoxygenation (Fig. 2A-B). Similar observations were also seen in rat cardiomyocytes of neonatal (nrCMs) or adult (arCMs) origins (Supplementary Fig. S4). These data suggest that spikebased vaccine has little effect on the survival of cultured cardiovascular cells even after hyp-reox injury.

To explore whether AdSpike affects hCM function in both normoxia and hyp-reox situations, we assessed spontaneous intracellular $\mathrm{Ca}^{2+}$ fluctuations in hCMs that infected by AdVector or AdSpike at various MOI using confocal laser microscopy. Individual hCM in each condition exhibited independent spontaneous beating and rhythmic $\mathrm{Ca}^{2+}$ transients. We found that the amplitude, time to peak, time of decay to $63 \%$ peak (T-63\% decay) of the $\mathrm{Ca}^{2+}$ transients were similar in the AdVector and AdSpike groups, whether they were subjected to hyp-reox injury or not (Fig. 2C; Supplementary Fig. S5A). Once again, we independently confirmed this conclusion in nrCMs (Supplementary Fig. S5B). To further assess the calcium handling properties, we subjected AdVector- or AdSpike-infected hCMs, nrCMs, and arCMs to a series of increasing electrical field stimulation frequencies. We found that AdSpike group could keep pace with the increasing stimulation under both normoxia or hyp-reox situations, similar to what was observed in the AdVector control. Moreover, no significant differences in the amplitude and rise/decay rates of $\mathrm{Ca}^{2+}$ transients were observed among the AdSpike groups and the AdVector groups with or without hyp-reox injury in all the three types of cardiomyocytes examined (Fig. 2D; Supplementary Fig. S6-7). The harmonic response of AdSpike-infected cardiomyocytes indicates that their calcium handling machinery could take up and release calcium stores in time for higher pulse stimulation frequencies. Thus, spike-based vaccine does not affect the calcium handling properties of cultured cardiomyocyte with or without hyp-reox injury.

To provide a further assessment of AdSpike on human heart tissue with or without ischemic injury, we took advantage of the human engineered heart tissue (hEHT) model (Fig. 3A; Supplementary Fig. S8) which widely expressed ACE2 (Fig. 3B). We infected the cells with AdSpike while fabricating the hEHTs (Fig. 3C; Supplementary Fig. S8A). The infection efficiency was very high as indicated by staining of the spike-fused flag epitopes (Fig. 3C), and no increased 
cell death was observed (shown by PI staining) (Fig. 3D; Supplementary Fig. S9A). As unhealthy tissues always show abnormal morphology during hEHT culturing [11], we thus monitored the morphology of hEHTs by examining their width. The width of hEHTs decreased along with culture time, indicating that the hEHTs were gradually getting matured (Supplementary Fig. S9B). We traced more than $103 \mathrm{hEHTs}$ for up to 10 days and compared the AdSpike-infected hEHTs with the AdVector control. Under both normoxia and hyp-reox conditions, we found that they exhibited similar width (Supplementary Fig. S9C) and spontaneous contraction properties, including amplitude and 50\% peak time, albeit there was a slight increase in beating rate in hEHTs infected by high MOI AdSpike (Fig. 3E; Supplementary Fig. S9D). Consistently, there was no difference between the AdVector and AdSpike groups in beating amplitude or 50\% beating peak time under $1.5 \mathrm{~Hz}$ electrical pacing (Fig. 3F; Supplementary Fig. S9E). More importantly, we used a customized contractility force test system to analyze the hEHT's contraction in stepped raising stretching length (stretching ratio 0\%, 2\%, 4\%, 6\%) [11] (Supplementary Fig. S8D). We observed that the contractility force increased while stretching in both AdVector and AdSpike hEHT groups, whereas there was no significant difference in max contractility force and max/min contractility force ratio between the two groups (Fig. 3G; Supplementary Fig. S9F). Similar results in contraction raising time were observed (Fig. 3G; Supplementary Fig. S9F). In aggregate, the spikeencoding vaccine does not affect hEHT function in both normoxia and hyp-reox situations.

To further explore the in vivo effects of spike-based vaccine on hearts after myocardial I/R injury, we intramuscular injected AdSpike to wild-type C57BL/6 mice at a dose that is equal $\left(1 \times 10^{9}\right.$ viral particles per kilogram of body weight, AdSpike-low $)$ or ten-fold higher $\left(1 \times 10^{10}\right.$ viral particles per kilogram of body weight, AdSpike-high) as used in human [12], subjected the mice to myocardial I/R injury 7 days post-vaccination, and examined the effects of AdSpike on heart function over a period of 28 days (Supplementary Fig. S10A). AdVector at a dose of $1 \times 10^{10}$ viral particles per kilogram of body weight was used as a control. I/R injury was created by temporal ligating the left anterior descending coronary artery in vivo for 1 hour followed by reperfusion. Effective immunogenicity of AdSpike at both high or low dose was confirmed by the specific 
ELISA antibody responses to the receptor binding domain (RBD) 28 days post-vaccination (Fig. 4A). By high-resolution echocardiography, we found that heart function was equally preserved after AdSpike vaccination compared to the AdVector control, reflected by the left ventricular ejection fraction and fractional shortening (Supplementary Fig. S10B). Consistently, we observed comparable scar sizes and heart weight/body weights between the vaccinated and control mice (Supplementary Fig. S10C-D). These data suggest that spike-based vaccine does not aggravate heart damage in wild-type mice after myocardial I/R injury.

It is known that the orthologous Ace 2 receptor in mice has a lower affinity to bind the spike protein, therefore typical inbred mouse strains do not support robust SARS-CoV-2 infection and replication [13]. To exclude the possibility that the above observation was an experimental artifact caused by the inefficient binding between spike and the mouse Ace2, we utilized a humanized mouse model in which the human ACE2 coding-sequence was knock-in into the mouse Ace2 genomic locus and replaced its mouse ortholog (Supplementary Fig. S10E-F). By using this model, we further confirmed that ACE2 expression was not significantly declined by AdSpike vaccination at both doses, whether subjected to myocardial I/R injury or not (Fig. 4B). In accordance with the observation in wild-type mice, histological and functional influences of AdSpike vaccination on I/R hearts of humanized ACE2 mice were neglectable when compared with the controls (Fig. 4CE). Furthermore, neither the number of $\mathrm{TUNEL}^{+}$apoptotic cardiomyocytes or vascular density in the border zone was altered by AdSpike vaccination (Fig. 4F). Taken together, spike-based vaccine has no effect on cardiomyocyte apoptosis, scar formation, infarct revascularization, and function recovery during myocardial I/R injury.

Hopes for a COVID-19 vaccine are now a reality. Although the dominant pathology of COVID-19 involves the respiratory system, 20\%-30\% of COVID-19 patients experience severe cardiovascular damage, which emerged as a major indicator of poor prognosis [8,14]. Moreover, patients with pre-existing heart complications are more likely to develop severe illness and have higher risk of death compared with patients without co-morbidities [14,15]. Therefore, a COVID19 vaccine would be lifesaving for patients with or at risk of heart diseases and warrants expedited 
vaccination. However, this group of people has been excluded from COVID-19 vaccine trials thus far and little is known about the performance of COVID-19 vaccines in them. Here, by combining models of human pluripotent stem cell-derived cardiac cells/tissues and humanized mouse models, we provide a proof-of-principle demonstration that spike-based Ad5 vaccine does not increase myocardial damage after ischemic injury. To our knowledge, this study represents the first systematic evaluation of the safety of a leading COVID-19 vaccine under a heart disease context. Although there are still many unanswered questions, we expect the spike-based COVID-19 vaccine to be safe and at least partly effective after myocardial ischemic injury, and benefits of vaccination likely outweigh risks of vaccine-related adverse events. This study may not only pave the way for clinical trials of people with cardiovascular conditions receiving these vaccines, but also provide an example that may be adapted for elucidate the safety and efficacy of COVID-19 vaccines in other complex diseases context. 


\section{SUPPLEMENTARY DATA}

Supplementary data are available online.

\section{ACKNOWLEDGEMENTS}

We thank professor Hui Zhang (Sun Yat-Sen University) for assistance in spike antibody titer determination.

\section{FUNDING}

N.C. is funded by the National Key R\&D Program of China (2018YFA0109100 and 2018YFA050830), the National Natural Science Foundation of China (92057113, 82061148011, and 31771508), and the Guangdong Innovative and Entrepreneurial Research Team Program (2016ZT06S029). D.Z. is funded by the National Natural Science Foundation of China (31871496) and the Hubei Science Fund for Distinguished Young Scholars (2019CFA092).

\section{AUTHOR CONTRIBUTIONS}

N.C., D.Z., and S.G. conceived the project and wrote the manuscript. S.G., Z.C., X.M., G.L., H.X. carried out the experiments. L.W., J.G., D.C., B.X., L.H., L.Z., Z.W., X.C., X.Z., and J.W. performed data analysis, provided materials, or assisted with the experiments.

Conflict of interest statement The authors declare no competing interests.

\section{REFERENCES}

1 Hoffmann M, Kleine-Weber H, Schroeder S et al. Cell 2020; 181: 271-80 e8.

2 Speiser D E \& Bachmann M F. Vaccines (Basel) 2020; 8:

3 Desai A, Gainor J F, Hegde A et al. Nat Rev Clin Oncol 2021;

$4 \quad$ Hwang J K, Zhang T, Wang A Z et al. J Hematol Oncol 2021; 14: 38.

$5 \quad$ Soltani S, Zandi M, Shiri Aghbash P et al. Biotechnol Appl Biochem 2020;

6 Turner A J, Hiscox J A \& Hooper N M. Trends Pharmacol Sci 2004; 25: 291-4.

7 Burrell L M, Risvanis J, Kubota E et al. Eur Heart J 2005; 26: 369-75; discussion 22-4. 
8 Nishiga M, Wang D W, Han Y et al. Nat Rev Cardiol 2020; 17: 543-58.

9 Oudit G Y, Kassiri Z, Jiang C et al. Eur J Clin Invest 2009; 39: 618-25.

10 Chen I Y, Chang S C, Wu H Y et al. J Virol 2010; 84: 7703-12.

11 Zhang D, Shadrin I Y, Lam J et al. Biomaterials 2013; 34: 5813-20.

12 Zhu F C, Guan X H, Li Y H et al. Lancet 2020; 396: 479-88.

13 Datta P K, Liu F, Fischer T et al. Theranostics 2020; 10: 7448-64.

14 Huang C, Wang Y, Li X et al. Lancet 2020; 395: 497-506.

15 Yiangou L, Davis R P \& Mummery C L. Stem Cell Reports 2021; 16: 385-97. 
A
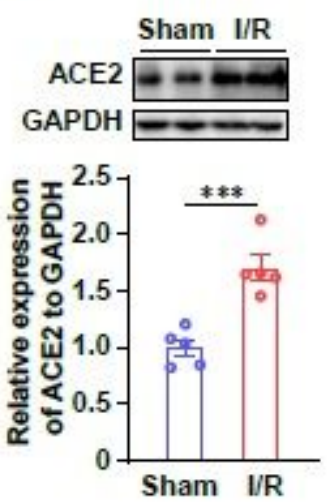

C
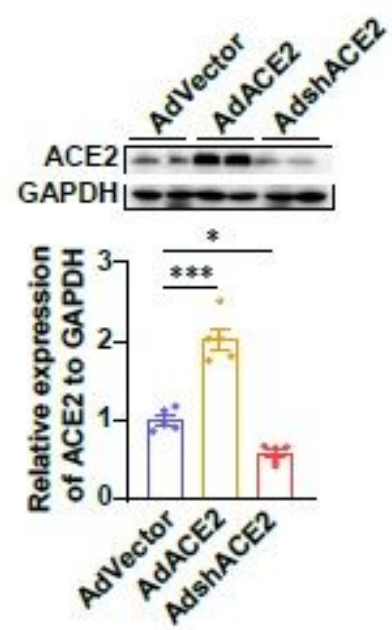

B

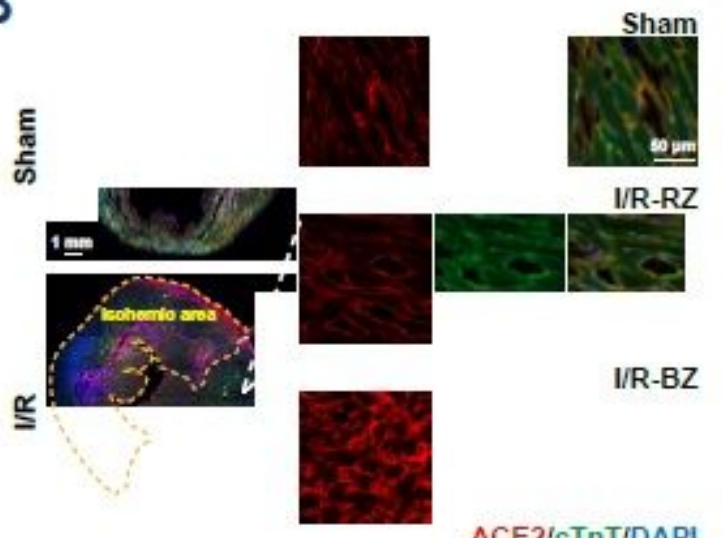

ACE2/cTnT/DAPI

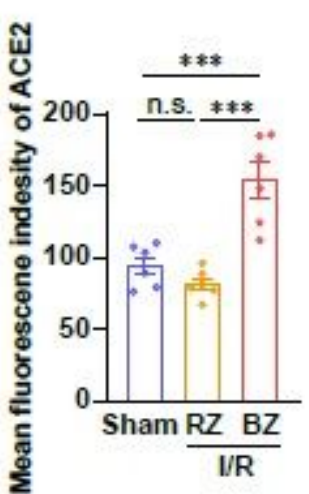

E

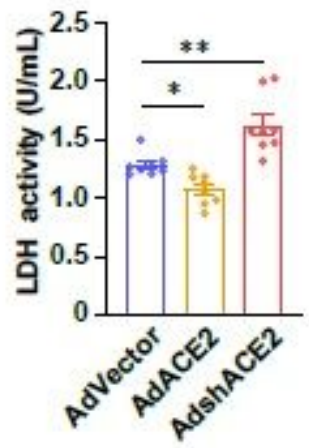

$\mathbf{F}$
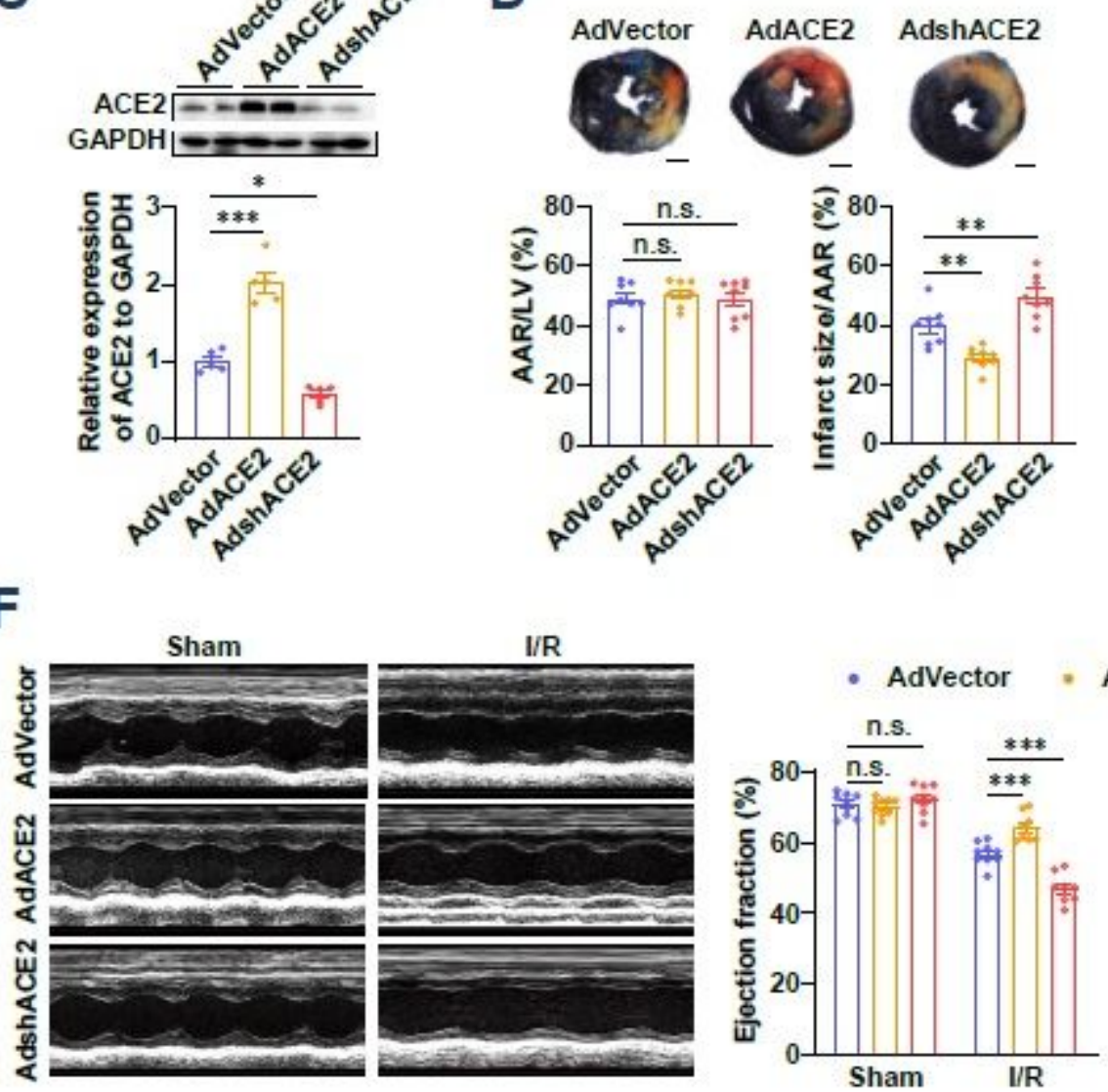

AdACE2 - AdshACE2

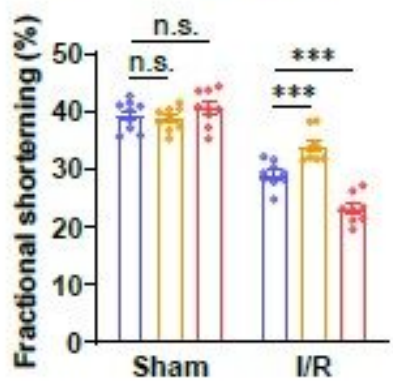

\section{Figure 1}

The protective effect of ACE2 during myocardial ischemia/reperfusion (I/R) injury. (A) Representative and quantitative immunoblot analysis of ACE2 protein in the hearts after myocardial I/R (45 min/24 h). $n=5$ for each group. (B) Representative immunostaining analysis of ACE2 expression with quantification of the mean fluorescence intensity in the hearts after myocardial I/R (45 min/24 h). RZ, remote zone; BZ, border zone. $n=6$ for each group. (C) Representative and quantitative immunoblot analysis of ACE2 protein in the hearts infected with adenovirus-mediated vector control (AdVector), adenovirus-mediated 
ACE2-overexpression (AdACE2) or adenovirus-mediated ACE2 short hairpin RNA (AdshACE2) for 3 days. $\mathrm{n}=5$ for each group. (D) Representative 2, 3, 5-triphenyltetrazolium chloride (TTC)/Evans blue staining (upper) and averaged infarct size (lower) following myocardial I/R (45 min/24 h). AAR, area at risk; LV, left ventricle; $n=8$ for each group; Scale bar, $1 \mathrm{~mm}$. (E) The lactic dehydrogenase (LDH) activity in the serum of mice infected with AdVector, AdACE2 or AdshACE2 following myocardial I/R ( 45 min/24 h). n=8 for each group. (F) Representative M-mode echocardiogram images and statistical analysis of ejection fraction and fractional shortening in the hearts infected with AdVector, AdACE2 or AdshACE2 following myocardial I/R (45 min/24 h). $n=8$ for each group. Data are presented as mean $\pm S E M ; * p<0.05,{ }^{\star *} p<$ $0.01, * \star \star p<0.001 ;$ n.s., not significant. 
A
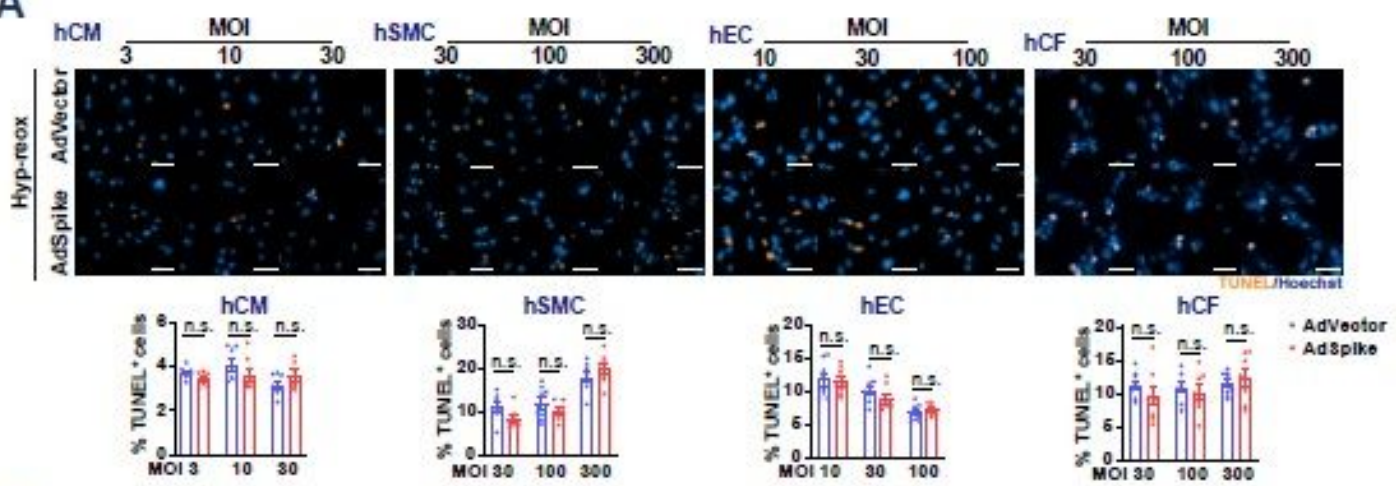

B
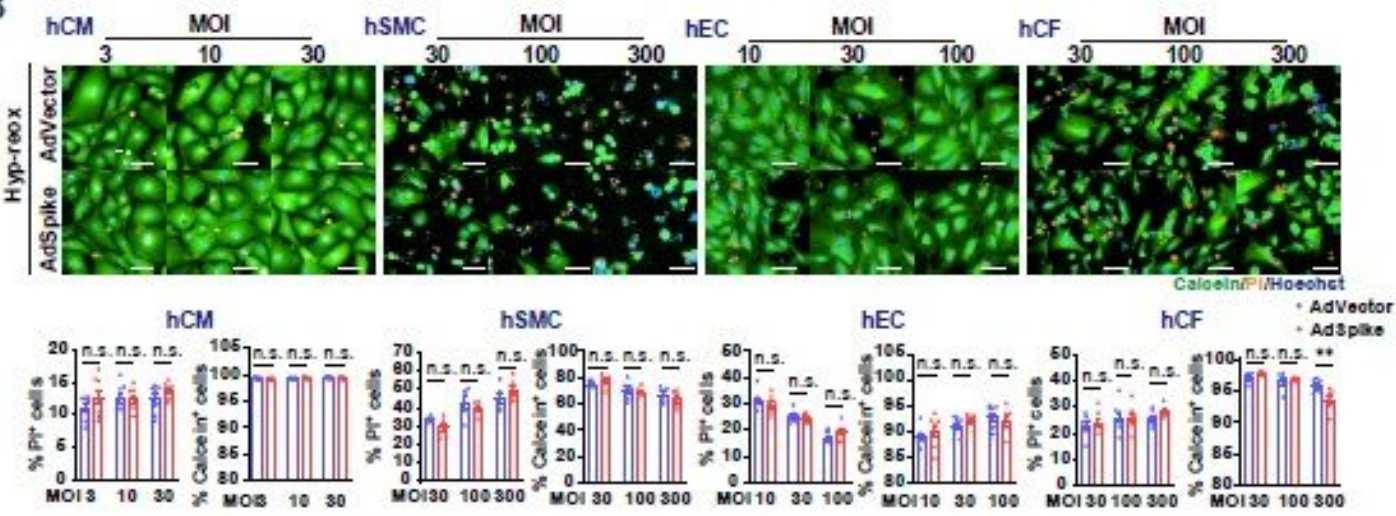

C
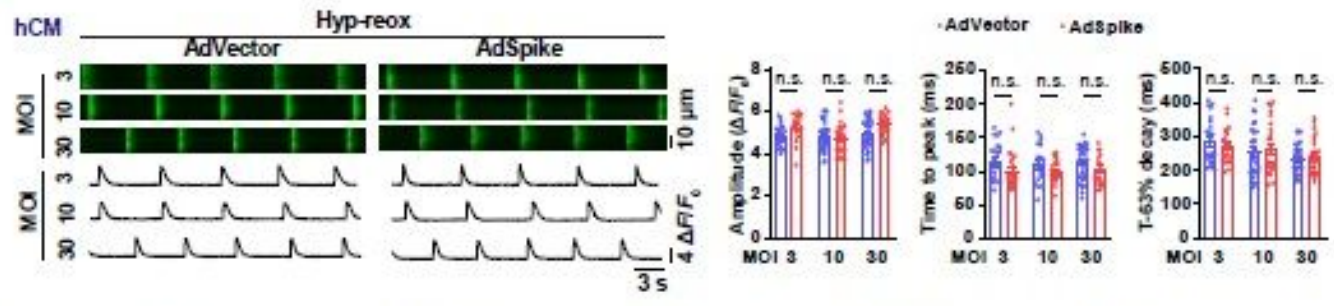

D
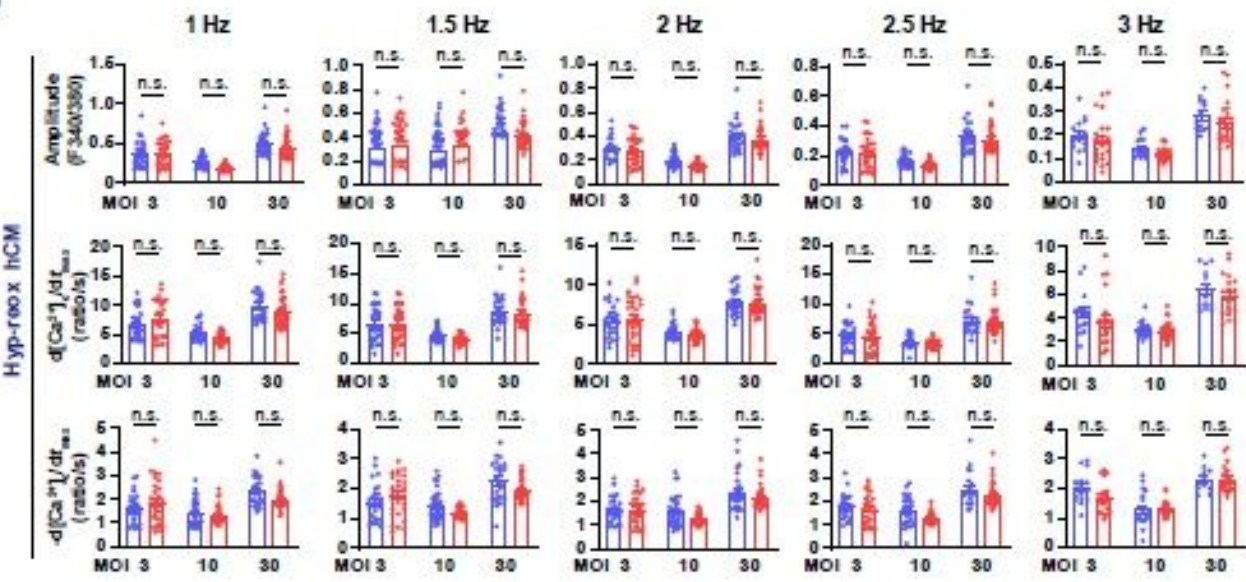

\section{Figure 2}

AdSpike has little effects on cell survival and function of cardiac cells following hypo-reox injury. (A) Representative and quantitative immunostaining analysis of the TUNEL+ cells in human cardiomyocytes (hCMs), human smooth muscle cells (hSMCs), human endothelial cells (hECs), and human cardiac fibroblasts (hCFs) infected with AdVector or AdSpike following hypoxia-reoxygenation (hyp-reox) injury. Scale bar, $50 \mu \mathrm{m}$. n=6-8 for each group. (B) Representative and quantitative calcein-AM/propidium iodide 
(PI) double staining in hCMs, hSMCs, hECs, hCFs infected by AdVector or AdSpike following hyp-reox injury. $n=6-8$ for each group. Scale bar, $50 \mu \mathrm{m}$. (C) Representative traces and averaged parameters of spontaneous intracellular calcium transient of hCMs infected with AdVector or AdSpike following hypreox injury. $n=15-30$ for each group. (D) Averaged parameters of calcium transient of the cardiomyocytes infected with AdVector or AdSpike with different frequency of electrical field stimulation. $n=15-30$ for each group. Data are presented as mean $\pm \mathrm{SEM}$; ${ }^{*} \mathrm{p}<0.01$; n.s., not significant.
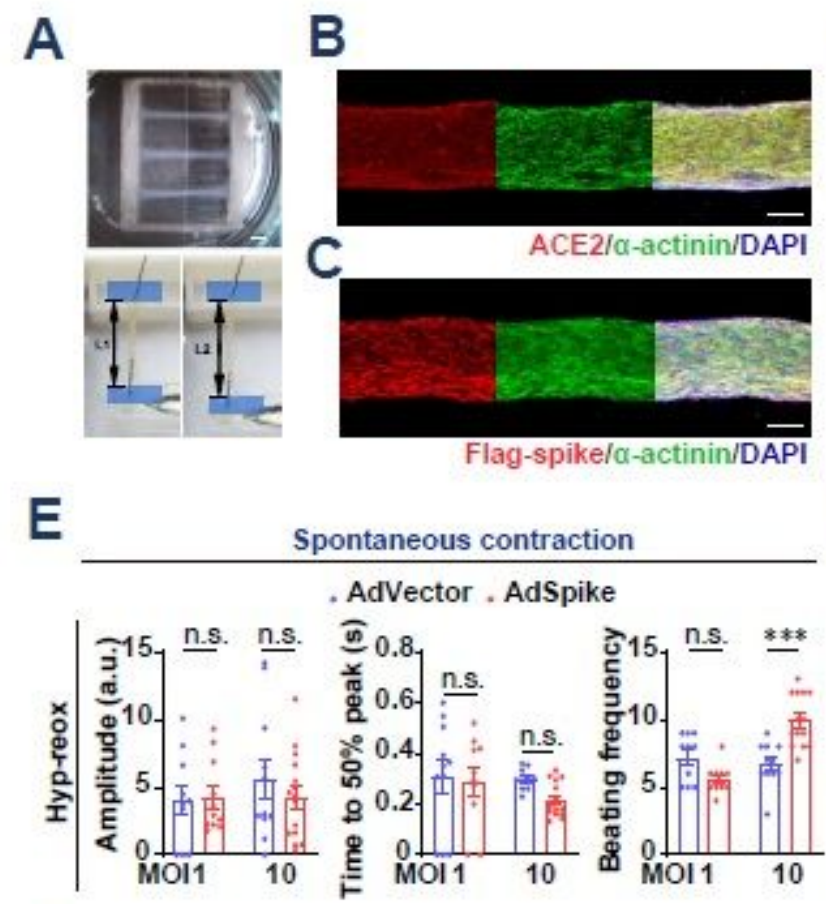

Spontaneous contraction
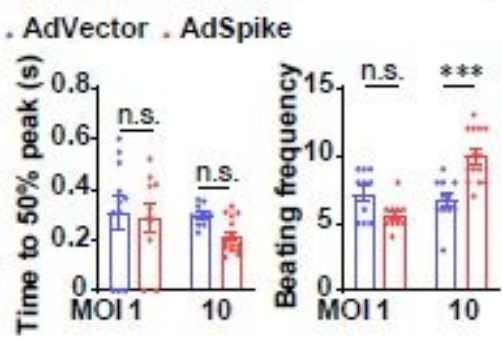
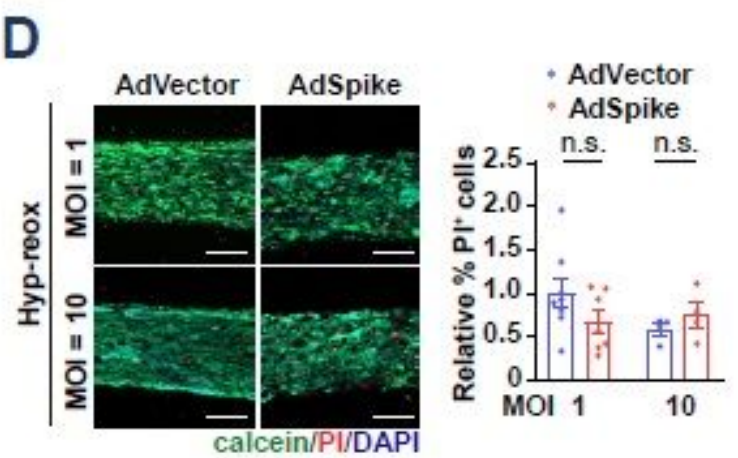

$\mathrm{F}$

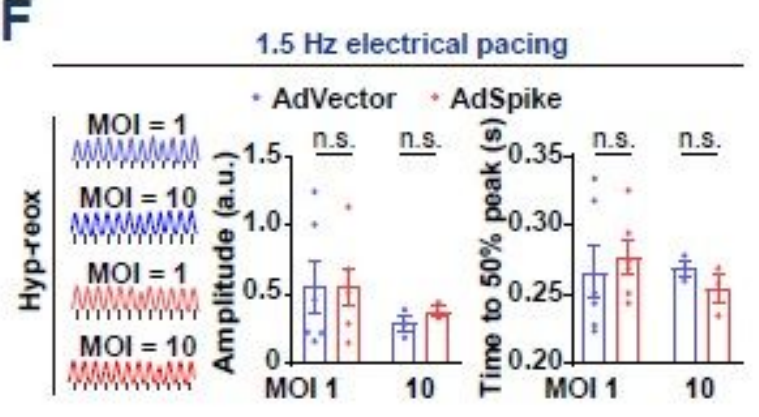

G
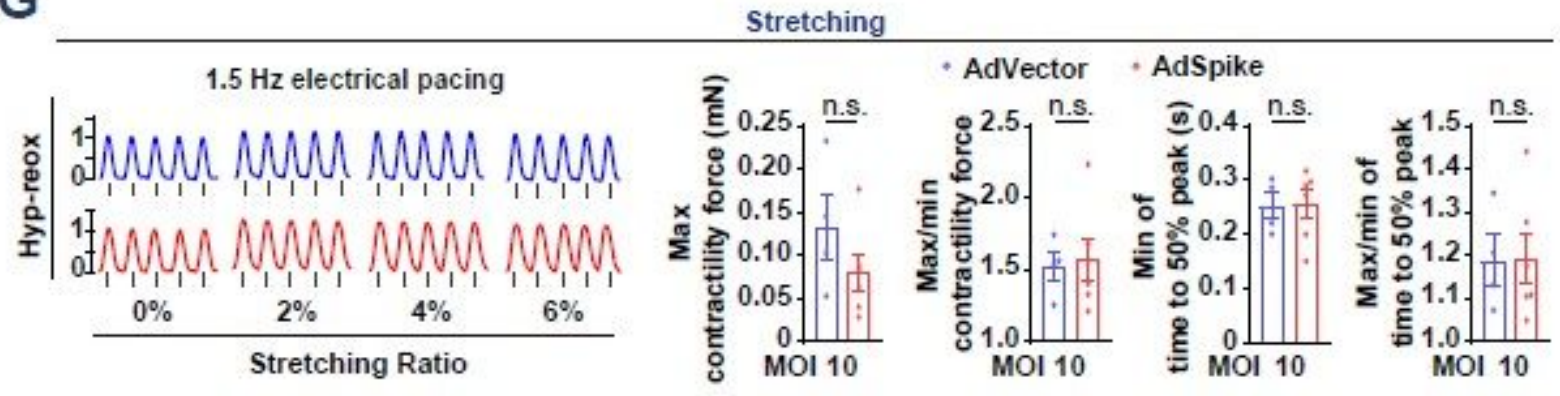

Figure 3

AdSpike has little effects on hEHTs that underwent spontaneous contraction, electrical field stimulation, and mechanical tensile test in hyp-reox condition. (A) Low magnification image of hEHT on day 21 (upper) and the equipment image of mechanical contractility force test for hEHT (lower). Scale bar, $2 \mathrm{~mm}$. (B) and (C). Co-localization analysis of anti-a-actinin (green) and anti-ACE2 (red) or anti-Spike-flag (red) with DAPI (blue). Scale bar, $200 \mu \mathrm{m}$. (D) Representative calcein-AM/PI double staining in hEHTs (left) with quantification (right). $n=4-8$ for each group. Scale bar, $200 \mu \mathrm{m}$. (E) Video analysis of the hEHTs for spontaneous contraction amplitude, time to $50 \%$ peak, and beating frequency per 10 seconds. a.u., absolute units. $n=7-17$ for each group. $(F)$ Representative contractile traces and contraction parameters' video analysis in $\mathrm{hEHTs}$ under $1.5 \mathrm{~Hz}$ electrical pacing. $n=3-6$ for each group. (G) Representative 
contractile force traces and contractile force parameters during progressive stretch (0\%, $2 \%, 4 \%, 6 \%$ tissue length) of hEHTs cultured in normoxia condition on day 24. $n=4$ for each group. All the hEHTs were infected with AdVector (MOI=1 or 10) or AdSpike (MOI=1 or 10) on day 14. Data are presented as mean \pm SEM; ${ }^{\star \star *} p<0.001$; n.s., not significant.

A

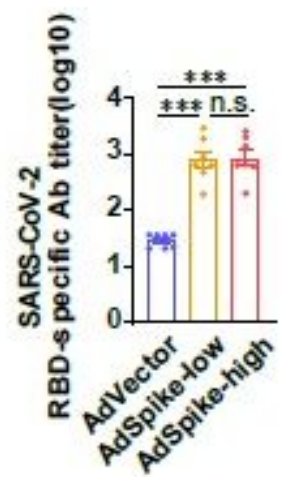

C
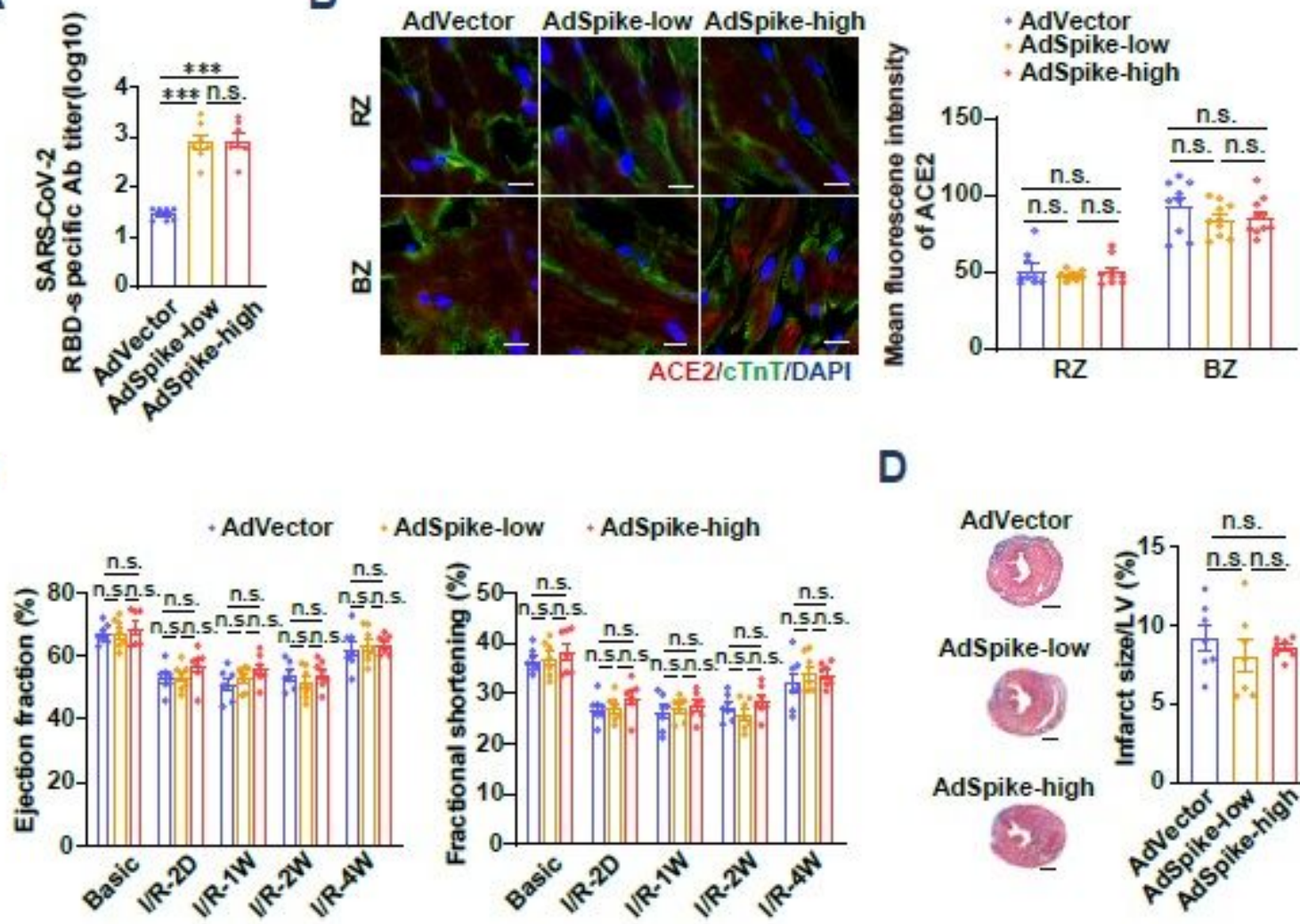

D

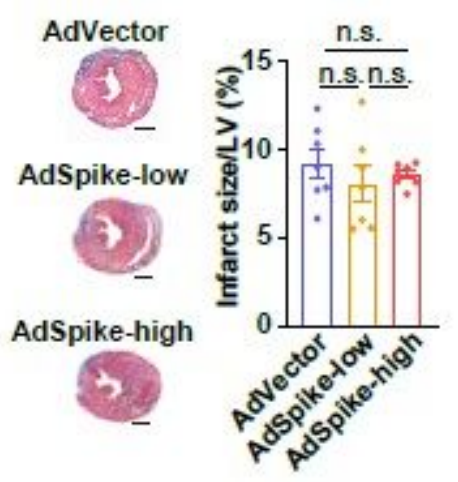

E

\section{$\mathrm{F}$}
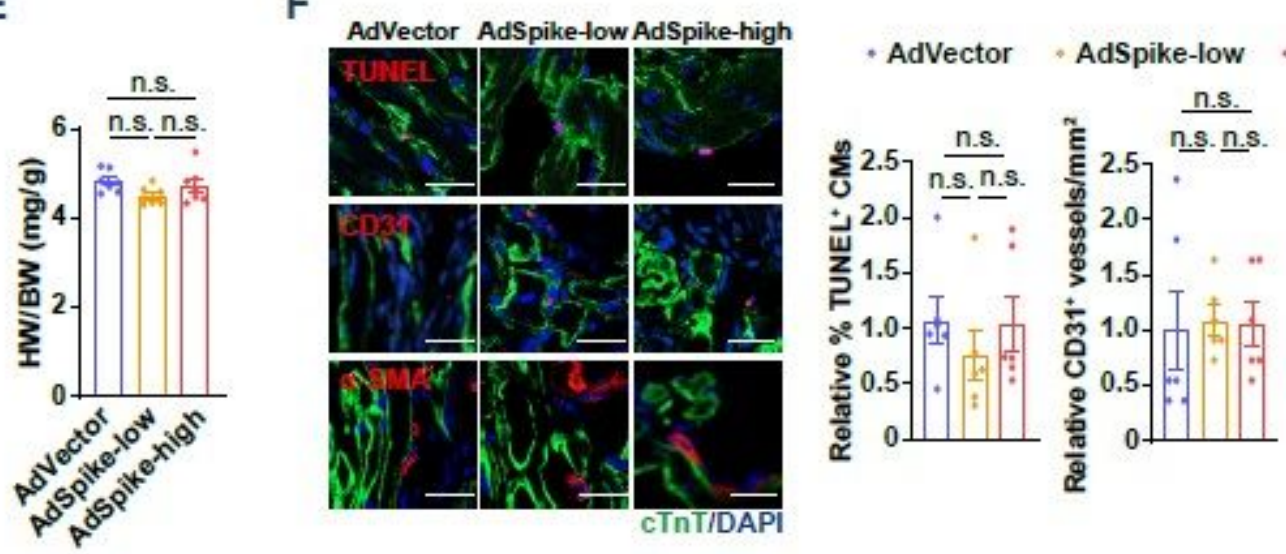

- AdSpike-high

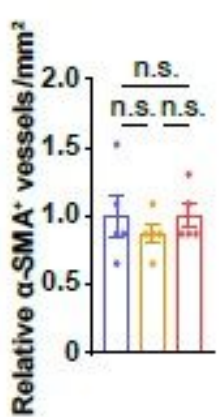

\section{Figure 4}

AdSpike has little effects on heart damage repair after myocardial I/R on humanized ACE2 (hACE2) mice in vivo. (A) Antibody tilters of SARS-CoV-2 spike receptor-binding domain (RBD) in the mouse serum collected at 4 weeks after injection of AdSpike at various doses. $n=7$ for each group. (B) Representative immunostaining analysis of the ACE2+ cardiomyocytes with quantification of the mean fluorescence intensity in border zone (BZ) or remote zone (RZ). $n=9$ for each group. Scale bar, $10 \mu \mathrm{m}$. (C) Ejection 
fraction and fractional shortening of hACE2 mice heart after I/R measured by echocardiographyat various time points post-I/R measured by echocardiography. $D$, day; W, week. $n=7$ for each group. (D) Masson-Trichrome staining of heart cross sections of hACE2 mice 4 weeks post-I/R (left) with quantification of scar size (right). $n=7$ for each group. Scale bar, $1 \mathrm{~mm}$. (E) Quantification of heart weight $(\mathrm{HW})$ relative to body weight (BW) of hACE2 mice at 4 weeks post-I/R. $n=7$ for each group. (F) Representative and quantitative immunostaining analysis of TUNEL+/CTnT+ cardiomyocytes, CD31+ capillaries, and a-SMA+ vessels in the border zones of infarcted hACE2 mouse hearts 4 weeks post-I/R. $n=5-6$ for each group. Scale bar, $50 \mu \mathrm{m}$. Data are presented as mean $\pm S E M ; * \star \star p<0.001 ; n$.s., not significant.

\section{Supplementary Files}

This is a list of supplementary files associated with this preprint. Click to download.

- Supplemental20210530.pdf 